\title{
3D Mathematical Model Characterizing the Dynamics of the Temperature Field of a Wall Structure with a Double-sided Facing from a Sapropel-hemp Composite Material
}

\author{
Sharif E. Guseynov \\ Faculty of Science and Engineering \\ Liepaja University \\ Liepaja, Latvia \\ sh.e.guseinov@inbox.lv
}

\author{
Aleksandrs Bereznojs \\ ISMA University of Applied Sciences \\ Riga, Latvia \\ alexander.v.berezhnoy@gmail.com
}

\author{
Janis S. Rimshans \\ Institute of Science and Innovative \\ Technologies \\ Liepaja University \\ Liepaja, Latvia \\ no email address
}

\author{
Stanislavs Pleiksnis \\ Faculty of Engineering \\ Rezekne Academy of Technologies \\ Rezekne, Latvia \\ stanislavs.pleiksnis@inbox.lv
}

\author{
Jekaterina V. Aleksejeva \\ Institute of Science and Innovative \\ Technologies \\ Liepaja University \\ Liepaja, Latvia \\ Riga Secondary School 34 \\ Riga, Latvia \\ jekaterina.v.aleksejeva@gmail.com
}

\begin{abstract}
In this paper, a 3D mathematical model is proposed to determine the dynamics of the temperature field in a three-layer composite sapropel-hemp slab. The proposed model consists of a system of three initial-boundary value problems with respect to the temperature function for each layer, respectively, and one initial-boundary value problem with respect to the unknown velocity of heat propagation along the thickness dimension of the composite sapropelhemp slab.
\end{abstract}

Keywords - Composite sapropel-hemp slab, temperature field, mathematical model

\section{INTRODUCTION}

One of the main ways to study the dynamics of the temperature field in multilayer building structures is the apparatus of the thermal conductivity theory, where the hypothesis of a continuous medium is used in modelling, which leads to obtaining linear and nonlinear differential equations: therefore, there appears smoothness requirement both in time and by spatial variables [1]-[3] related to the functions characterizing the properties and states of the components of the medium.
The main goal of such studies is to find the temperature field inside a composite body under known initial and boundary conditions [4]. In this paper, we consider the problem of determining the temperature field in a threelayer composite slab of the parallelepiped shape, where the outer layers (fireproof facing) are bonded with hemp and sapropel mineralized fill. Since sapropel consists mainly of organic substances (ash content of organo-mineral sapropel varies within $25 \div 50 \%$, mineral-organic - 50-70\%, mineralized - $70 \div 85 \%$, organic - about $30 \%$ ), then over time, the temperature state of the sapropel-hemp slab will significantly depend, in addition to its heat exchange with the environment, also on the processes of heat release/heat absorption by the volume of the slab, happening due to various reasons (it must be taken into account that the viscosity of the sapropel can reduce gas exchange): for example, resulting in exothermic/endothermic chemical reactions [5]; the energy of radiation penetrating into the volume of the slab through the deformed facing [3],[6],[7]; etc. Consequently, inside the sapropel-hemp slab, internal sources of thermal energy are formed, which can have various types of dependences both on time and spatial variables, as well as on the temperature itself: for example, 
if the energy release in the inner layer of a sapropel-hemp slab is caused by chemical reactions, which rate is described by the Arrhenius equation, then the dependency of the volumetric power of energy release on temperature has a reverse exponential character [5],[8].

In this work, a 3D mathematical model is proposed that describes dynamics of the temperature field in the studied sapropel-hemp slab. In the proposed mathematical model, due to the specifics of the material of the inner layer sapropel-hemp material, it is assumed that, firstly, the temperature conductivity coefficient is a given step function along the vertical axis directed along the thickness dimension of the sapropel-hemp slab, and secondly, the velocity of heat propagation, along with the thermal field, is also considered to be the required function.

\section{RESTRICTIVE ASSUMPTIONS, AND CONSTRUCTION OF MATHEMATICAL MODEL FOR TEMPERATURE FIELD}

Before proceeding to the description of the mathematical model, let us choose the Cartesian coordinate system so that the positive semiaxis of the vertical axis $\mathrm{OX}_{3}$, characterizing the thickness of the considered sapropel-hemp slab is directed from the outer facing of the slab to the inner side.

Fig. 1 schematically depicts the studied composite sapropel-hemp slab having the size (length, width, height/thickness, respectively) with a double-sided fireproof facing: the outer facing $P_{L_{1}, L_{2}, L_{3}}^{0,0,0}$, where $P_{b_{1}, b_{2}, \cdots, b_{n}}^{a_{1}, a_{2}, \ldots, a_{n}} \equiv\left\{x \in \mathbb{R}^{n}: x_{i} \in\left[a_{i}, b_{i}\right], i=\overline{1, n}\right\}$, has a height $l_{1}$; the inner fireproof facing $P_{x_{1}, x_{2}, L_{3}}$ has a height $L_{3}-l_{2}$; the sapropel-hemp component (i.e. the second/inner layer) of the composite slab has a height $l_{2}-l_{1}$.

Now we can proceed to the construction of a mathematical model for the dynamics of the temperature field of the considered sapropel-hemp slab, having previously listed its physical and mathematical peculiarities and the necessary mathematical assumptions arising from these aspects [9]-[12]. Due to the fact that the considered composite slab is a solid non-periodic heterogeneous medium, the following three circumstances will be present in the constructed 3D mathematical model of the temperature field dynamics [8], [13]-[19]:

First, it cannot be assumed that the velocity of heat propagation is a priori given. Obviously, this circumstance also gives rise to the necessity of constructing a mathematical model to determine the unknown heat propagation velocity. Consequently, there is a need to construct a complete mathematical model consisting of two interconnected equations with the corresponding initial and boundary conditions: in the first equation with respect to the desired unsteady temperature field $T(x, t)$, there is a vector function

$$
\vec{\vartheta} \stackrel{\text { def }}{\equiv} \vec{\vartheta}(x, t)=\left(\vartheta_{x_{1}}, \vartheta_{x_{2}}, \vartheta_{x_{3}}\right)^{T}(x, t),
$$

describing the velocity of heat propagation in directions $O X_{1}, O X_{2}, O X_{3}$, and the second equation is an equation relating to this unknown velocity.

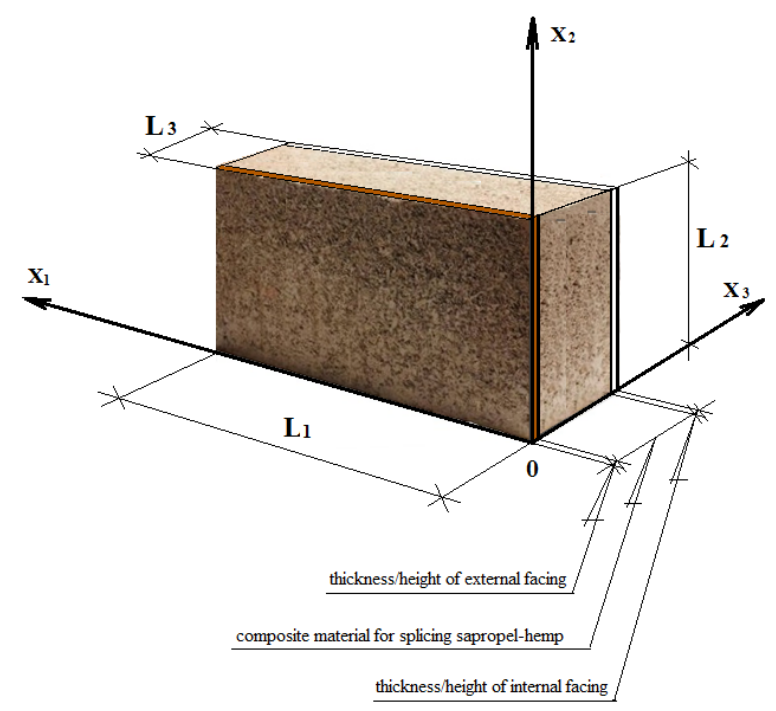

Fig. 1. Composite sapropel-hemp slab.

Second, the coefficient of temperature conductivity $\alpha\left(\vec{\vartheta}_{T}\right)$ can be considered a step function along the vertical axis $\mathrm{OX}_{3}$ (i.e. relating to the layers). This circumstance, together with the assumption about the insignificance of thermal perturbations [13] in directions $O X_{1}$ and $O X_{2}$, allows to construct a one-dimensional by the spatial variable (i.e. in the vertical component) nonlinear inhomogeneous equation with respect to the heat propagation averaged over directions $O X_{1}$ and $O X_{2}$ vector velocity in a composite sapropel-hemp slab, i.e. with respect to scalar function $\vartheta_{x_{3}}(x, t)$.

Third, due to the fact that the first and third layers interacting with the environment (i.e. facing - outer layers) of the considered composite slab are made of homogeneous fireproof materials (for example, made of clay; of mineral fibres; etc., moreover, it is not necessary that both facings are made of the same fireproof material), and the second layer (inner layer) is its sapropel-hemp component (solid non-periodic heterogeneous medium), then from a mathematical point of view the heating processes on the first and third layers should proceed with the heat flux relaxation mode [17]-[19].

Taking into account the corresponding basic fundamental laws of physics - the laws of molecular diffusion and the laws of heat transfer in solid non-periodic heterogeneous media [13]-[15], [20], - taking into account the above listed three circumstances, we will have the following mathematical model: for $\forall t \geq 0$ it is required to determine the temperature field 


$$
T(x, t)=\left\{\begin{array}{l}
T^{\{1\}}(x, t), x \in P_{L_{1}, L_{2}, l_{1}}^{0,0,0}, \\
T^{\{2\}}(x, t), x \in P_{L_{1}, L_{2}, l_{2}}^{0,0, l_{1}}, \\
T^{\{3\}}(x, t), x \in P_{L_{1}, L_{2}, L_{3}}^{0,0, l_{2}},
\end{array}\right.
$$

at any point $x \in P_{L_{1}, L_{2}, L_{3}}^{0,0,0}$ of the composite sapropel-hemp slab from

- a heterogeneous partial differential equation:

$$
\begin{aligned}
& \frac{\partial T^{\{1\}}(x, t)}{\partial t}+R_{1} \frac{\partial^{2} T^{\{1\}}(x, t)}{\partial t^{2}}-T_{\text {external }}(x, t) \\
& =\operatorname{div}\left(\alpha(\vec{\vartheta}) \overrightarrow{\operatorname{grad}} T^{\{1\}}(x, t)\right), x \in \operatorname{int} P_{L_{1}, L_{2}, l_{1}}^{0,0,0} ;
\end{aligned}
$$

- two initial conditions:

$$
\begin{gathered}
\left.T^{\{1\}}(x, t)\right|_{t=0}=T_{0}^{\{1\}}(x), x \in P_{L_{1}, L_{2}, l_{1}}^{0,0,0} ; \\
\left.\frac{\partial T^{\{1\}}(x, t)}{\partial t}\right|_{t=0}=T_{1}^{\{1\}}(x), x \in P_{L_{1}, L_{2}, l_{1}}^{0,0,0} ;
\end{gathered}
$$

- six boundary conditions:

- Neumann homogeneous boundary condition at the right end of the outer facing:

$$
\frac{\partial T^{\{1\}}(x, t)}{\partial x_{1}}=0, x \in P_{0, L_{2}, l_{1}}^{0,0,0}\left(x_{2}, x_{3}\right),
$$

- Neumann homogeneous boundary condition at the left end of the outer facing:

$$
\frac{\partial T^{\{1\}}(x, t)}{\partial x_{1}}=0, x \in P_{L_{1}, L_{2}, l_{1}}^{L_{1}-0,0}
$$

- Neumann homogeneous boundary condition at the underside of the outer facing:

$$
\frac{\partial T^{\{1\}}(x, t)}{\partial x_{2}}=0, x \in P_{L_{1}, 0, l_{1}}^{0,0,0},
$$

- Neumann homogeneous boundary condition at the upper side of the outer facing:

$$
\frac{\partial T^{\{1\}}(x, t)}{\partial x_{2}}=0, x \in P_{L_{1}, L_{2}, l_{1}}^{0, L_{2}-0,0},
$$

- Robin boundary condition at the outer surface of the outer facing:

$$
\begin{aligned}
& -\chi(\vec{\vartheta}) \frac{\partial T^{\{1\}}(x, t)}{\partial x_{3}}+\beta_{1}^{m_{1}}\left\{T^{\{1\}}(x, t)\right. \\
& \left.+\theta_{\text {external }}(t)\right\}^{m_{1}}=0, x \in P_{L_{1}, L_{2}, 0}^{0,0,0},
\end{aligned}
$$

- Dirichlet inhomogeneous boundary condition on the inner surface of the outer facing:

$$
T^{\{1\}}(x, t)=T^{\{2\}}\left(x_{1}, x_{2}, t\right), x \in P_{L_{1}, L_{2}, l_{1}}^{0,0, l_{1}-0} ;
$$

- two conjugation conditions:

$$
\begin{array}{r}
\left.T^{\{1\}}(x, t)\right|_{x_{3}=l_{1}-0}=\left.T^{\{2\}}(x, t)\right|_{x_{3}=l_{1}+0}, x \in P_{L_{1}, L_{2}}^{0,0}, \\
\left.\left\{\alpha(\vec{\vartheta}) \frac{\partial T^{\{1\}}(x, t)}{\partial x_{3}}\right\}\right|_{x_{3}=l_{1}-0}, \\
=\left.\left\{\alpha(\vec{\vartheta}) \frac{\partial T^{\{2\}}(x, t)}{\partial x_{3}}\right\}\right|_{x_{3}=l_{1}+0}, x \in P_{L_{1}, L_{2}}^{0,0} ;
\end{array}
$$

- corresponding consistency constraints of the initial functions from (3), (4) and boundary functions from (5)-(10);

- heterogeneous partial differential equation:

$$
\begin{aligned}
& \frac{\partial T^{\{2\}}(x, t)}{\partial t}=\operatorname{div}\left(\alpha(\vec{\vartheta}) \cdot \overrightarrow{\operatorname{grad}} T^{\{2\}}(x, t)\right) \\
& -\vec{\vartheta}_{T}(x, t) \cdot \overrightarrow{\operatorname{grad}} T^{\{2\}}(x, t) \\
& +T^{\{1\}}(x, t)+T^{\{3\}}(x, t), x \in \operatorname{int} P_{L_{1}, L_{2}, l_{2}}^{0,0, l_{1}} ;
\end{aligned}
$$

- one initial condition:

$$
\left.T^{\{2\}}(x, t)\right|_{t=0}=T_{0}^{\{2\}}(x), x \in P_{L_{1}, L_{2}, l_{2}}^{0,0, l_{1}} ;
$$

- six boundary conditions:

- Dirichlet inhomogeneous boundary condition at the right end of the sapropel-hemp material:

$$
T^{\{2\}}(x, t)=T_{\text {right }}^{\{2\}}\left(x_{2}, x_{3}, t\right), x \in P_{0, L_{2}, l_{2}}^{0,0, l_{1}},
$$

- Dirichlet inhomogeneous boundary condition at the left end of the sapropel-hemp material:

$$
T^{\{2\}}(x, t)=T_{\text {left }}^{\{2\}}\left(x_{2}, x_{3}, t\right), x \in P_{L_{1}, L_{2}, l_{2}}^{L_{1}-0,0, l_{1}},
$$

- Dirichlet inhomogeneous boundary condition at the underside of the sapropel-hemp material:

$$
T^{\{2\}}(x, t)=T_{\text {inter. }}^{\{2\}}\left(x_{1}, x_{3}, t\right), x \in P_{L_{1}, 0, l_{2}}^{0,0, l_{1}},
$$

- Dirichlet inhomogeneous boundary condition at the upper side of the sapropel-hemp material:

$$
T^{\{2\}}(x, t)=T_{\text {exter. }}^{\{2\}}\left(x_{1}, x_{3}, t\right), x \in P_{L_{1}, L_{2}, l_{2}}^{0, L_{2}-0, l_{1}},
$$

- Robin boundary condition at the surface of the sapropel-hemp material that is adjacent to the inner surface of the outer facing:

$$
\begin{aligned}
& h \frac{T^{\{2\}}(x, t)-T^{\{1\}}(x, t)}{\chi\left(\vartheta_{x_{3}}\right)} \\
& =\frac{\partial T^{\{2\}}(x, t)}{\partial x_{3}}, x \in P_{L_{1}, L_{2}, l_{1}}^{0,0, l_{1}},
\end{aligned}
$$

- Robin boundary condition at the surface of the sapropel-hemp material, which is adjacent to the inner surface of the inner facing: 
Sharif E. Guseynov, et al. 3D Mathematical Model Characterizing the Dynamics of the Temperature Field of a Wall Structure with a Double-Sided Facing from a Sapropel-Hemp Composite Material

$$
\begin{aligned}
& h \frac{T^{\{1\}}(x, t)-T^{\{2\}}(x, t)}{\chi\left(\vartheta_{x_{3}}\right)} \\
& =\frac{\partial T^{\{2\}}(x, t)}{\partial x_{3}}, x \in P_{L_{1}, L_{2}, l_{2}}^{0,0, l_{2}-0} ;
\end{aligned}
$$

- four conjugation conditions:

$$
\begin{aligned}
& \left.T^{\{2\}}(x, t)\right|_{x_{3}=l_{1}+0}=\left.T^{\{1\}}(x, t)\right|_{x_{3}=l_{1}-0}, x \in P_{L_{1}, L_{2}}^{0,0}, \\
& \left.\left\{\alpha(\vec{\vartheta}) \frac{\partial T^{\{2\}}(x, t)}{\partial x_{3}}\right\}\right|_{x_{3}=l_{1}+0} \\
& =\left.\left\{\alpha(\vec{\vartheta}) \frac{\partial T^{\{2\}}(x, t)}{\partial x_{3}}\right\}\right|_{x_{3}=l_{1}-0}, x \in P_{L_{1}, L_{2}}^{0,0}, \\
& \left.T^{\{2\}}(x, t)\right|_{x_{3}=l_{2}-0}=\left.T^{\{3\}}(x, t)\right|_{x_{3}=l_{2}+0}, x \in P_{L_{1}, L_{2}}^{0,0}, \\
& \left.\left\{\alpha(\vec{\vartheta}) \frac{\partial T^{\{2\}}(x, t)}{\partial x_{3}}\right\}\right|_{x_{3}=l_{2}-0} \\
& =\left.\left\{\alpha(\vec{\vartheta}) \frac{\partial T^{\{3\}}(x, t)}{\partial x_{3}}\right\}\right|_{x_{3}=l_{2}+0}, x \in P_{L_{1}, L_{2}}^{0,0} ;
\end{aligned}
$$

- corresponding consistency constraints of the initial function from (14) and boundary functions from (15), (16);

- heterogeneous partial differential equation:

$$
\begin{aligned}
& \frac{\partial T^{\{3\}}(x, t)}{\partial t}+R_{2} \frac{\partial^{2} T^{\{3\}}(x, t)}{\partial t^{2}}-T_{\text {inter. }}(x, t) \\
& =\operatorname{div}\left(\alpha(\vec{\vartheta}) \cdot \overrightarrow{\operatorname{grad}} T^{\{3\}}(x, t)\right), x \in \operatorname{int} P_{L_{1}, L_{2}, L_{3}}^{0,0, l_{2}} ;
\end{aligned}
$$

- two initial conditions:

$$
\begin{gathered}
\left.T^{\{3\}}(x, t)\right|_{t=0}=T_{0}^{\{3\}}(x), x \in P_{L_{1}, L_{2}, L_{3}}^{0,0, l_{2}}, \\
\left.\frac{\partial T^{\{3\}}(x, t)}{\partial t}\right|_{t=0}=T_{1}^{\{3\}}(x), x \in P_{L_{1}, L_{2}, L_{3}}^{a_{1}, a_{2}, l_{2}}
\end{gathered}
$$

- six boundary conditions:

- Neumann homogeneous boundary condition at the right end of the inner facing:

$$
\frac{\partial T^{\{3\}}(x, t)}{\partial x_{1}}=0, x \in P_{0, L_{2}, L_{3}}^{0,0, l_{2}},
$$

- Neumann homogeneous boundary condition at the left end of the inner facing:

$$
\frac{\partial T^{\{3\}}(x, t)}{\partial x_{1}}=0, x \in P_{L_{1}, L_{2}, l_{1}}^{L_{1}-0,0,0},
$$

- Neumann homogeneous boundary condition at the underside of the inner facing:

$$
\frac{\partial T^{\{3\}}(x, t)}{\partial x_{2}}=0, x \in P_{L_{1}, 0, L_{3}}^{0,0, l_{2}},
$$

- Neumann homogeneous boundary condition at the upper side of the inner facing:

$$
\frac{\partial T^{\{3\}}(x, t)}{\partial x_{2}}=0, x \in P_{L_{1}, L_{2}, L_{3}}^{a_{1}, L_{2}-0, l_{2}},
$$

- Dirichlet inhomogeneous boundary condition at the inner surface (that is, the surface that is adjacent to the second layer) of the inner facing:

$$
T^{\{3\}}(x, t)=T^{\{2\}}\left(x_{1}, x_{2}, t\right), x \in P_{L_{1}, L_{2}, l_{2}}^{0,0, l_{2}},
$$

- Robin boundary condition at the outer surface of the inner facing:

$$
\begin{aligned}
& -\chi(\vec{\vartheta}) \frac{\partial T^{\{3\}}(x, t)}{\partial x_{3}}+\beta_{2}^{m_{2}}\left\{T^{\{3\}}(x, t)\right. \\
& \left.-\theta_{\text {inter. }}(t)\right\}^{m_{2}}=0, x \in P_{L_{1}, L_{2}, L_{3}}^{0,0, L_{3}-0} ;
\end{aligned}
$$

- two conjugation conditions:

$$
\begin{array}{r}
\left.T^{\{3\}}(x, t)\right|_{x_{3}=l_{2}+0}=\left.T^{\{2\}}(x, t)\right|_{x_{3}=l_{2}-0}, x \in P_{L_{1}, L_{2}}^{0,0}, \\
\left.\left\{\alpha(\vec{\vartheta}) \frac{\partial T^{\{3\}}(x, t)}{\partial x_{3}}\right\}\right|_{x_{3}=l_{2}+0}, x \in P_{L_{1}, L_{2}, b_{3}}^{0,0} ;
\end{array}
$$

- from the corresponding consistency constraints of the initial functions from (3), (4) and boundary functions from (5)-(10).

Before proceeding to the description and explanation of the meaning and purpose of the designations that take place in (2)-(35), let us note the following specifics of the mathematical model (2)-(35).

First peculiarity. Obviously, (2)-(12) is a mathematical model for finding a function $T^{\{1\}}(x, t)$, defined only on the first layer (i.e. on the outer facing) of a composite sapropel-hemp slab. Note that this mathematical model includes an unknown function (see (10)-(12)), defined only on the second layer (i.e. on the inner layer - in the sapropel-hemp component) of the composite slab. This means that without knowing the function $T^{\{2\}}(x, t)$, it is impossible to determine the required function $T^{\{1\}}(x, t)$ from (2)-(12) 
Environment. Technology. Resources. Rezekne, Latvia Proceedings of the $13^{\text {th }}$ International Scientific and Practical Conference. Volume 3, 95-103

Second peculiarity. (24)-(34) is a mathematical model for finding a function $T^{\{3\}}(x, t)$, defined only on the third layer (i.e. on the inner facing) of a composite slab. As in model (2)-(12), this mathematical model includes the same unknown function $T^{\{2\}}(x, t)$ (see (32), (34), (35)). This means that, without knowing the function $T^{\{2\}}(x, t)$, it is impossible to determine the desired function $T^{\{3\}}(x, t)$ from (25)-(35).

Third peculiarity. (13)-(24) is a mathematical model for determining the function $T^{\{2\}}(x, t)$, defined only on the second layer (i.e. on the inner layer - in the sapropelhemp part) of the composite slab. This model includes (see (13), (19)-(24)) both a function $T^{\{1\}}(x, t)$, defined only on the first layer and a function $T^{\{3\}}(x, t)$, defined only on the third layer. This means that without knowing the functions $T^{\{1\}}(x, t)$ and $T^{\{3\}}(x, t)$, it is impossible to determine the desired function $T^{\{3\}}(x, t)$ from (13)-(24).

From the above listed peculiarities of the mathematical model (2)-(35), it becomes clear that this model (2)-(35) consists of three independent mathematical models interconnected by their sought functions $T^{\{1\}}(x, t)$, $T^{\{2\}}(x, t), \quad T^{\{3\}}(x, t)$ : of the mathematical model (2)(12); mathematical model (13)-(24); mathematical model (25)-(35). As a rule, such a situation arises in those studied real problems of mathematical physics, in which the objects under study are multilayer objects or the investigated processes and phenomena occur in layered media [13], [22], [23]. When studying and solving mathematical models of this kind, describing real processes, phenomena or objects in many fields of science and technology, it is imperative to deal with a special class of problems in the theory of ordinary differential equations, called the Sturm-Liouville problem [24], the analysis and solution of which is often a rather cumbersome process and requires from the researcher either special knowledge in mathematics [13], [22]-[24], or the involvement of a professional mathematician in the research, who's scientific interests cover both the theory of differential equations and mathematical physics.

Now let us briefly describe the meaning and purpose of the designations in model (2)-(35).

(A) First of all, we note that in the mathematical model (2)-(35), the sought functions are only two functions: function (1), which characterizes the unsteady temperature field in all three layers of the composite sapropel-hemp slab; function $\vec{\vartheta}(x, t)$, that characterizes the vector velocity of heat propagation in a composite sapropel-hemp slab in directions $O X_{1}, O X_{2}, O X_{3}$.

(B) Mathematical model (2)-(35) does not contain a separate sub model for determining the required function $\vec{\vartheta}(x, t)$ : in the next section, a separate mathematical model will be formulated (see (36)-(47)) to determine this function, or rather, to determine the heat propagation vector velocity averaged by directions $O X_{1}$ and $O X_{2}$ in a composite sapropel-hemp slab, i.e. to define a scalar function $\vartheta_{x_{3}}(x, t)$ (see the second peculiarity at the beginning of this section).

(C) $\operatorname{div} \vec{A}(x, t) \stackrel{\text { def }}{\equiv} \sum_{j=1}^{3} \frac{\partial A_{x_{j}}(x, t)}{\partial x_{j}}$, called the divergence operator, is a differential operator that maps a vector field $\vec{A}(x, t)$ to a scalar field. At each fixed moment in time, the operator $\operatorname{div} \vec{A}(x, t)$ for each spatial point $x \in \mathbb{R}^{3}$ determines how much the incoming and outgoing heat fluxes diverge. In the mathematical model (2)-(35), the operator $\operatorname{div}(\bullet)$ is present in equations (2), (13) and (25) with vector fields $\vec{A}=\alpha(\vec{\vartheta}(x, t)) \overrightarrow{\operatorname{grad}} T^{\{j\}}(x, t)$, $j=\overline{1,3}$, where $\overrightarrow{\operatorname{grad}} T^{\{j\}}(x, t)$, the called gradient of a scalar function $T^{\{j\}}(x, t),(j=\overline{1,3})$ is a vector whose coordinates are the partial derivatives of this scalar function $T^{\{j\}}(x, t)$. In the mathematical model (2)-(35), the gradient $\overrightarrow{\operatorname{grad}}(\bullet)$ is present in equations (2), (13), and (25) with scalar functions $T^{\{1\}}(x, t), \quad T^{\{2\}}(x, t)$ and $T^{\{3\}}(x, t)$, respectively.

(D) $R_{1}>0$ from equation (2) and $R_{2}>0$ from equation (24) are referred to as the heat flux relaxation coefficients in the outer facing (i.e. the first layer) and the inner facing (i.e. the third layer), respectively. These numerical coefficients are assumed to be known, for example, being determined experimentally. Further, $\beta_{1}>0$ from the boundary condition (9) and from the boundary condition (33) characterize the averaged heat transfer property of the outer and inner facings, respectively. These numerical parameters are also assumed to be known. Further, $m_{1}>0$ from the boundary condition (9) and $m_{2}>0$ from the boundary condition 
(33) characterize the diffusion properties of the outer and inner facings, respectively. These numerical values are also assumed to be known. Further, the meaning of positive numbers $L_{1}, L_{2}, L_{3}, l_{1}$ and $l_{2}$ is quite obvious from Fig. 1. All these numbers are obviously assumed to be known.

(E) All initial and boundary functions are assumed to be given a priori: initial functions $T_{0}^{\{1\}}(x), x \in P_{L_{1}, L_{2}, L_{3}}^{0,0,0}$, $T_{1}^{\{1\}}(x), x \in P_{L_{1}, L_{2}, L_{3}}^{0,0,0}, \quad T_{0}^{\{2\}}(x), x \in P_{L_{1}, L_{2}, l_{2}}^{0,0, l_{1}}$, $T_{0}^{\{3\}}(x), P_{L_{1}, L_{2}, L_{3}}^{0,0, l_{2}}, \quad T_{1}^{\{3\}}(x), x \in P_{L_{1}, L_{2}, L_{3}}^{0,0, l_{2}} ; \quad$ boundary functions $T_{\text {right }}^{\{2\}}\left(x_{2}, x_{3}, t\right), x_{2} \in\left[0, L_{2}\right], x_{3} \in\left[l_{1}, l_{2}\right], t \geq 0$, $T_{\text {left }}^{\{2\}}\left(x_{2}, x_{3}, t\right), x_{2} \in\left[0, L_{2}\right], x_{3} \in\left[l_{1}, l_{2}\right], t \geq 0$, $T_{\text {inter. }}^{\{2\}}\left(x_{1}, x_{3}, t\right), x_{1} \in\left[0, L_{1}\right], x_{3} \in\left[l_{1}, l_{2}\right], t \geq 0$, $T_{\text {exter. }}^{\{2\}}\left(x_{1}, x_{3}, t\right), x_{1} \in\left[0, L_{1}\right], x_{3} \in\left[l_{1}, l_{2}\right], t \geq 0 ; \quad$ functions $\theta_{\text {exter. }}(t), t \geq 0, \quad \theta_{\text {inter. }}(t), t \geq 0$, meaning the ambient temperature from which the surfaces of the outer and inner facings perform heat exchange according to Newton's law, respectively [13]; functions $T_{\text {exter. }}(x, t), x \in \operatorname{int} P_{L_{1}, L_{2}, l_{1}}^{0,0,0}$, $T_{\text {inter. }}(x, t), x \in \operatorname{int} P_{L_{1}, L_{2}, L_{3}}^{0,0, l_{2}}$, which characterize the density of heat sources in the environment, with which heat exchange occurs through the surfaces of the outer and inner facings, respectively.

(F) $\alpha(\vec{\vartheta}(x, t))=\frac{\chi(\vec{\vartheta}(x, t))}{c_{p} \rho(\vec{\vartheta}(x, t))}$ is the temperature conductivity coefficient of a composite sapropel-hemp slab, where $\chi\left(\vartheta_{x_{3}}\right)$ denotes the thermal conductivity coefficient, $\rho\left(\vartheta_{x_{3}}\left(x_{3}, t\right)\right)$ denotes density, $c_{p}$ denotes the specific heat capacity. Due to three peculiarities that were presented at the beginning of this section, in the mathematical model (2)-(35) this temperature conductivity coefficient is assumed to be a known step function for $\forall x_{i} \in\left[0, L_{i}\right]$, $(i=1,2)$ :

$$
\begin{aligned}
& 0<\alpha_{\min } \leq \alpha(\vec{\vartheta}(x, t)) \approx \alpha\left(\vartheta_{x_{3}}\left(x_{3}, t\right)\right) \\
& =\left\{\begin{array}{l}
\alpha_{1} \text { if } 0 \leq x_{3} \leq l_{1}, \\
\alpha_{2} \text { if } l \leq x_{3} \leq l_{2}, \\
\alpha_{3} \text { if } l_{2} \leq x_{3} \leq L_{3},
\end{array}\right.
\end{aligned}
$$

where $\alpha_{i}, i=\overline{1,3}$ are the constants.

\section{MATHEMATICAL MODEL FOR DESIRED VECTOR VELOCITY OF HEAT PROPAGATION}

As it has been mentioned in the previous section, the function $\vec{\vartheta}(x, t)$ characterizes the vector velocity of heat propagation in a composite sapropel-hemp slab along the directions $O X_{1}, O X_{2}, O X_{3}$. Assuming that the velocity of heat propagation along the axis $\mathrm{OX}_{3}$ (i.e. along the thickness/height dimension of the sapropel-hemp slab) is much higher than the velocity of heat propagation in the other two directions (i.e. along the width and length dimensions of the sapropel-hemp slab), then the vector velocity $\vec{\vartheta}(x, t)$ can be averaged along the direction of the $O X_{1}$ and $O X_{2}$ axes. Then, instead of the vector of the function $\vec{\vartheta}(x, t)$, you can consider only its third component $\vartheta_{x_{3}}(x, t)$. In this section, a mathematical model is proposed for finding this particular scalar function.

So, let us turn to the formulation of a mathematical model for a function $\vartheta_{x_{3}}\left(x_{3}, t\right)$, that is present in many places of the mathematical model (1)-(34), but still remains undefined.

It is required to define a function $\vartheta_{x_{3}}\left(x_{3}, t\right), x_{3} \in\left[0, L_{3}\right]$ for $\forall t \geq 0$ from the following one nonlinear equation, one initial condition, four boundary conditions, two conjugation conditions, and four consistency constraints:

- heterogeneous partial differential equation:

$$
\begin{aligned}
& \frac{\partial \vartheta_{x_{3}}\left(x_{3}, t\right)}{\partial t}=\frac{\partial}{\partial x_{3}}\left(\alpha\left(\vartheta_{x_{3}}\left(x_{3}, t\right)\right) \frac{\partial \vartheta \vartheta_{x_{3}}\left(x_{3}, t\right)}{\partial x_{3}}\right) \\
& -\vartheta_{x_{3}}\left(x_{3}, t\right) \frac{\partial \vartheta_{x_{3}}\left(x_{3}, t\right)}{\partial x_{3}}+P\left(x_{3}, t\right), 0<x_{3}<L_{3} ;
\end{aligned}
$$

- initial condition:

$$
\left.\vartheta_{x_{3}}\left(x_{3}, t\right)\right|_{t=0}=\vartheta_{0}\left(x_{3}\right), 0 \leq x_{3} \leq L_{3} ;
$$

- boundary conditions:

- Dirichlet inhomogeneous boundary condition:

$$
\left.\vartheta_{x_{3}}\left(x_{3}, t\right)\right|_{x_{3}=0+0}=\theta_{\text {exter. }}(t),
$$

- Neumann homogeneous boundary condition:

$$
\left.\frac{\partial \vartheta_{x_{3}}\left(x_{3}, t\right)}{\partial x_{3}}\right|_{x_{3}=l_{1}-0}=0
$$

- Neumann homogeneous boundary condition:

$$
\left.\frac{\partial \vartheta_{x_{3}}\left(x_{3}, t\right)}{\partial x_{3}}\right|_{x_{3}=l_{2}+0}=0
$$

- Dirichlet inhomogeneous boundary condition: 
Environment. Technology. Resources. Rezekne, Latvia Proceedings of the $13^{\text {th }}$ International Scientific and Practical Conference. Volume 3, 95-103

$$
\left.\vartheta_{x_{3}}\left(x_{3}, t\right)\right|_{x_{3}=L_{3}-0}=\theta_{\text {inter. }}(t)
$$

- conjugation conditions:

$$
\begin{gathered}
\left.\vartheta_{x_{3}}\left(x_{3}, t\right)\right|_{x_{3}=l_{1}-0}=\left.\vartheta_{x_{3}}\left(x_{3}, t\right)\right|_{x_{3}=l_{1}-0}, t \geq 0, \\
\left.\left\{\alpha\left(\vartheta_{x_{3}}\left(x_{3}, t\right)\right) \frac{\partial \vartheta_{x_{3}}\left(x_{3}, t\right)}{\partial x_{3}}\right\}\right|_{x_{3}=l_{2}-0} \\
=\left.\left\{\alpha\left(\vartheta_{x_{3}}\left(x_{3}, t\right)\right) \frac{\partial \vartheta_{x_{3}}\left(x_{3}, t\right)}{\partial x_{3}}\right\}\right|_{x_{3}=l_{2}-0} ;
\end{gathered}
$$

- consistency constraints of the initial function from (37) and boundary functions from (38)-(41):

$$
\left.\begin{array}{l}
\vartheta_{x_{3}}(0+0)=\theta_{\text {exter. }}(0+0), \\
\vartheta_{x_{3}}\left(L_{3}-0\right)=\theta_{\text {inter. }}(0+0), \\
\vartheta_{x_{3}}^{\prime}\left(l_{1}-0\right)=\vartheta_{x_{3}}^{\prime}\left(l_{2}+0\right)=0 .
\end{array}\right\}
$$

The function $P\left(x_{3}, t\right), x_{3} \in\left(0, L_{3}\right), t \geq 0$, characterizes the total density of heat sources of the environment external to the composite sapropel-hemp slab. In the mathematical model (36)-(44), all the initial data have already been described in the frames of the mathematical model (2)-(35).

So, the complete mathematical model proposed in this work consists of two mathematical models (listed in the sequence of the solution required): from the mathematical model (36)-(44) with respect to the function $\vartheta_{x_{3}}\left(x_{3}, t\right)$ of the averaged velocity of heat propagation, and the mathematical model (2)-(35) with respect to the desired unsteady temperature field $T(x, t)=T\left(x_{1}, x_{2}, x_{3}, t\right)$ of composite sapropel-hemp slab.

If to find a solution to model (36)-(44), the equation of which can be attributed to the class of equations of the Fisher-Kolmogorov-Petrovsky-Piskunov type [25]-[27], then finding a solution to model (2)-(35) will not cause particular difficulties: this can be implemented by the separating variables method (also known as the Fourier method) [6], [13], [22], [23]. In this paper, we are not dealing with the solution of the one-dimensional initialboundary value problem (36)-(44), but here we would only like to note that equation (36) using a nondegenerate transformation

$$
\left\{\begin{array}{l}
\vartheta_{x_{3}}\left(x_{3}, t\right)=\frac{\partial W\left(x_{3}, t\right)}{\partial x_{3}} \\
w\left(x_{3}, t\right)=\ln \left[U\left(x_{3}, t\right)\right]^{-\alpha\left(\vartheta_{x_{3}}\left(x_{3}, t\right)\right)}
\end{array}\right.
$$

is reduced to a linear equation relating to the new sought for function $U\left(x_{3}, t\right)$. The resulting initial-boundary value problem can also be solved by the separating variables method.

\section{DisCUSSION}

The nomenclature of composite materials used in construction is constantly expanding, and innovative methods are developed that make it possible to improve the physical-mechanical, thermophysical, chemical, environmental, economic, etc. characteristics of materials: for example, resulting in substantial improvement of hydrophobicity, fire resistance, intensity of heat exchange processes, statistical and dynamic strength, endurance, energy efficiency, sound insulation, etc. One of the most beneficial from the environmental friendliness and costeffectiveness standpoint within the nomenclature of the composite construction panels is considered to be a slab made of the hemp shives (acting as filler) and sapropel (acting as binding). Sapropels are a complex organic or organomineral complex of various chemical compounds, both preserved in the original biomass and newly formed in the process of biochemical metamorphism of organic substances [28]. In Latvia, the processes of their formation and accumulation are constantly ongoing, and for many water reservoirs these processes become progressive [28]. In Latvia, the processes of its formation and accumulation are ongoing, and for many freshwater lakes (over 3 thousand with an area larger than 1 ha) and swamps (over 6 thousand) [29]-[31], which are under the control of the "Latvian state forests" governmental institution, these processes are having a progressive nature: according to preliminary estimates, these reservoirs contain over 3 billion $\mathrm{m}^{3}$ of sapropel. Due to the high degree of destruction of fragments of the original biomass, sapropel are characterized by a homogeneous structure with a high content of fine-grained particles and a high water-holding capacity.

Getting back to the constructed mathematical model (2)-(44), let us note that knowledge of the thermal field inside of a three-layer composite sapropel-hemp slab allows us to calculate (at least in first approximation) the strength of the enclosing and supporting structures built of such slabs under thermal loading conditions: specifically, the temperature changes are the root cause for appearing of additional strain in the structure [32]-[34]. However, in our opinion, in order to carry out an adequate strength calculation for the constructions under thermal loading conditions, it is necessary to consider the mathematical model (2)-(44) in a many component system: this is especially necessary if a construction built of composite sapropel-hemp slabs, refers to: (1) objects of landscape architecture; (2) objects of household facilities in rural areas; (3) self-contained buildings; (4) sites in an urban canyon-like area; (5) objects under the influence of 
periodic or almost periodic aero-, hydro-, or lithodynamic flows [34]-[36]. The abovementioned means that the corresponding boundary conditions in (2)-(35) must be "stitched" at the left $P_{L_{1}, L_{2}, L_{3}}^{L_{1}, 0,0}$ and right $P_{0, L_{2}, L_{3}}^{0,0,0}$ ends of each of two adjacent sapropel-hemp slabs. Since the construction consists, as a rule, of a multiple sapropelhemp slabs, it seems that the specified "stitching" of the boundary conditions for each adjacent pair of slabs will significantly complicate the mathematical model (2)-(35) within its computer modeling implementation. In fact, if we consider the mathematical model (2)-(35) in a system of only two bodies/slabs and "stitching" the corresponding boundary conditions to carry out between the right $P_{0, L_{2}, L_{3}}^{0,0,0}$ end of the left adjacent slab and the left $P_{L_{1}, L_{2}, L_{3}}^{L_{1}, 0,0}$ end of the right neighboring slab, then this approach will be quite acceptable averaging of the mentioned complex system consisting of many bodies/slabs: there is no need to apply other types of averaging (for instance, see fundamental monograph [34] as well as [37]).

\section{CONCLUSIONS}

In this paper, a 3D mathematical model is proposed to determine the dynamics of the temperature field in a threelayer composite sapropel-hemp parallelepiped slab, in which the outer layers (fireproof facing) are bonded with hemp and sapropel mineralized fill. In the proposed mathematical model, due to the specifics of the material of the inner layer - sapropel-hemp material, it is assumed that, firstly, the temperature conductivity coefficient is a given step function along the vertical axis directed along the thickness dimension of the sapropel-hemp slab, and secondly, the velocity of heat propagation, along with the thermal field, is also considered to be the sought for function. The proposed model consists of a system of three initial-boundary value problems with respect to the temperature function for each layer, respectively, and one initial-boundary value problem with respect to the unknown velocity of heat propagation along the thickness dimension of the composite sapropel-hemp slab.

As it has been already mentioned at the end of Section III, the constructed model (2)-(44) can be solved by the method of separation of variables, by applying it separately to (2)-(35) and to (36)-(44) (to be more precise, to the linear initial-boundary value problem obtained after using the nondegenerate transformation (45)): we managed to find an analytical solution of the model (2)(44) in a closed form, however, unfortunately, we have not yet managed to implement it in a computer-based model and carry out the corresponding numerical experiments.

\section{ACKNOWLEDGEMENTS}

This work is dedicated to the blessed memory of Professor Janis S. Rimshans, Liepaja University.

The authors of this work would like to express their gratitude to the anonymous reviewers for their equitable critical comments and valuable advices, which we took into account with gratitude.

\section{REFERENCES}

[1] P. Germain, Cours de mécanique des milieux continus: Théorie générale. Paris: Masson et Cie, Éditeurs, 1973.

[2] Yu. I. Burchakov, V. E. Gnedin, and V. M. Denisov, Structural mechanics. Moscow: Higher School, 1983.

[3] A. V. Lykov, Heat conduction theory. Moscow: Higher School, 1967.

[4] R. I. Nigmatulin, Continuum mechanics: Kinematics, dynamics, thermodynamics, statistical dynamics. Moscow: GEOTAR-Media, 2014.

[5] D. A. Frank-Kamenetsky, Diffusion and heat transfer in chemical kinetics. Moscow: Nauka, 1987.

[6] H. S. Carslaw and J. C. Jaeger, Conduction of Heat in Solids. Oxford: Oxford University Press, 1986.

[7] V. S. Zarubin, Modelling. Moscow: "Academy" Publishing Centre, 2013.

[8] V. S. Zarubin, A. V. Kotovich, and G. N. Kuvyrkin, "Temperature state of a plate with temperature-dependent thermal conductivity and energy-release," Mathematics and Mathematical Modeling, vol. 16, no. 3, pp. 18-28, 2016.

[9] V. V. Vasiliev, Mechanics of structures made of composite materials. Moscow: Mechanical Engineering, 1988.

[10] L. N. Tyalina, A. M. Minaev, and V. A. Pruchkin, New composite cmaterials. Tambov: Tambov State Technical University Press, 2011.

[11] M. L. Kerber, Polymer composite materials: Structure, properties, technology. St. Petersburg: Profession, 2008.

[12] V. R. Bensman, "Classification problems for peat bogs and sapropels," Young scientist, vol. 1, no. 1-2, pp. 146-147, 2010.

[13] A. N. Tikhonov and A. A. Samarskii, Equations of Mathematical Physics. Moscow: Nauka, 1977.

[14] Th. L. Bergman, A. S. Lavine, F. P. Incropera, and D. P. Dewitt, Fundamentals of Heat and Mass Transfer. New Jersey: John Wiley \& Sons, 2011.

[15] J. H. Lienhard IV and J. H. Lienhard V, A Heat Transfer Textbook. - Massachusetts, Cambridge: Phlogiston Press, 2019.

[16] Y. Ashida, Ke. Saito, and M. Ueda, "Thermalization and heating dynamics in open generic many-body systems," Physical Review Letters, vol. 121, no. 7, pp. 402-408, 2018.

[17] P. P. Volosevich and E. I. Levanov, "Analysis of heat transfer processes taking into account the relaxation of heat flux and bulk energy sources in the medium," Transactions of Russian Higher Educational Institutions: Mathematics, vol. 47, no. 1, pp. 36-42, 2003.

[18] E. I. Levanov, "Heat transfer taking into account the relaxation of the heat flux and energy sources," Mathematical Modelling, vol. 9, no. 2, pp. 53-56, 1997.

[19] D. S. P. Salazar, A. M. S. Macedo, and G. L. Vasconcelos, "Quantum heat distribution in thermal relaxation processes," Physical Review E, vol. 99, no. 2, pp. 2133-2138, 2019.

[20] R. P. Feynman, R. B. Leighton, and M. Sands, The Feynman lectures on physics: Mainly mechanics, radiation, and heat. California: California Institute of Technology Press, 1963. 
[21] K. Micadei, J. P. S. Peterson, A. M. Souza, R. S. Sarthour, I. S Oliveira, G. T. Landi, T. B. Batalhao, R. M. Serra, and E. Lutz, "Reversing the direction of heat flow using quantum correlations," Nature Communications, vol. 10, paper no. 2456, 6 p, 2019.

[22] Sh. E. Guseynov, R. Aleksejevs, J. V. Aleksejeva, "On a 3D initialboundary value problem for determining the dynamics of impurities concentration in a horizontal layered fine-pore medium," in Proceedings of the 12th International Scientific and Practical Conference "Environment. Technology. Resources", vol. III, Rezekne: Rezekne Academy of Technologies Press, 2019, pp. 6069.

[23] Sh. E. Guseynov, R. Aleksejevs, J. V. Aleksejeva, and J. S. Rimshans, "On the impurities concentrations dynamics in multicomponent semidisperse semicolloid-high-molecular system: a case study of two-layered anisotropic peat block," Advanced Mathematical Models \& Applications, vol.4, no. 2, pp. 99-126, 2019.

[24] A. Zettl, Sturm-Liouville Theory. Providence, Rhode Island: American Mathematical Society Press, 2005.

[25] A. N. Kolmogorov, I. G. Petrovsky, and N. S. Piscounov, "Investigation of the diffusion equation combined with an increase in matter and its application to a biological problem," Bulletin of Moscow State University, Series A: Mathematics and Mechanivs, vol. 1, pp. 1-25, 1937.

[26] R. A. Fisher, "The wave of advance of advantageous genes," Annals of Eugenics, vol. 7, pp. 355-369, 1937.

[27] M.El-Hachem, S.W.McCue, W.Jin, Y.Du, and M.J.Simpson, "Revisiting the Fisher-Kolmogorov-Petrovsky-Piskunov equation to interpret the spreading-extinction dichotomy," Proceedings of the Royal Society A: Mathematical, Physical and Engineering Sciences, vol. 475, no. 2229, Paper ID: 0378, 2019.
[28] S. M. Shtin, Lacustrine sapropels and their complex development. Moscow State Mining University Press, 2005.

[29] N. Brakss, B. Vimba, and A. Kalnins, "On the properties and ways of using sapropels of the Latvian SSR," Transactions of Latvian Academy of Sciences, vol. 133, no. 8, pp. 43-50, 1958.

[30] Central Statistical Bureau of Latvia, "Geographical data," 2009. [Online].

Available: https://data.stat.gov.lv/pxweb/lv/OSP PUB/START ENV DR DRT/DRT020/. [Accessed: March 19, 2021].

[31] Database, "Lakes," $2009 . \quad$ [Online]. Available: https://www.ezeri.lv/?l=en. [Accessed: March 19, 2021].

[32] R. M. Christensen, Mechanics of composite materials. Mineola: Dover Publications, 2005.

[33] G. L. Gorynin and Yu. V. Nemirovsky, "Mathematical modelling of heat conduction process for 2D-periodic anisotropic composite materials," Mathematical Methods and Physico-Mechanical Field, vol. 57, no. 2, pp. 142-151, 2014.

[34] N. S. Bakhvalov and G. P. Panasenko, Averaging processes in periodic media. Mathematical problems in the mechanics of composite materials. Moscow: Science 1984.

[35] L. C. King, The morphology of the Earth: A study and synthesis of world scenery. Edinburgh: Oliver \& Boyd, 1962.

[36] I. P. Baranov, "Using the concept of plastics relief in solving problems modern city planning and architectural planning," in International Conference "InterCarto. InterGIS. Geoinformation Support of Sustainable Development of Territories", vol. 20, 2014, pp. 575-587.

[37] V. I. Gorbachev, "The Bakhvalov-Pobedri averaging method in the mechanics of composites," Bulletin of Lomonosov Moscow State University, Series 1: Mathematics and Mechanics, no. 6, pp. 41-45, 2016. 